\title{
Information und Verkehr
}

Verkehr - ein allgegenwärtiges Thema, das uns täglich in unseren Entscheidungen beeinflusst, vor allem auch durch negative Meldungen in den Medien.

Doch Mobilität von Personen und Gütern ist ein Schlüsselfaktor einer prosperierenden und wettbewerbsfähigen Volkswirtschaft, welche von den Mobilitätsbedürfnissen der Einzelpersonen und von den Anforderungen der weltweit vernetzten Industrie, der Arbeitsteiligkeit der industriellen Prozesse und den daraus resultierenden enorm gestiegenen Güterströmen geprägt ist.

Hunderte Millionen Fahrzeuge (auf Straße, Schiene, in Luft und auf Wasser) müssen allein in Europa täglich entlang einer Verkehrsinfrastruktur bewegt werden, deren Kapazitätsgrenzen vielfach schon erreicht scheinen.

Unfälle, Staus, Luftverschmutzung, Lärm und die Einschränkung der Lebensqualität sowie die daraus resultierenden Folgeerscheinungen verursachen einen volkswirtschaftlichen Schaden, der gemäß einer Studie der Europäischen Kommission allein in Europa mit $300 \mathrm{Mrd}$. Euro jährlich beziffert wird.

Intelligente Fahrzeuge und eine effiziente Verkehrssteuerung, die eine ausgewogene Nutzung der vorhandenen Infrastruktur und eine Verkehrsentwicklung unter dem Gesichtspunkt ökonomischer Wettbewerbsfähigkeit und ökologischer Verträglichkeit gewährleisten, sind ein Gebot der Stunde - nicht nur für die Verkehrspolitik in Europa.

Dem Einsatz der Informationstechnik zur Entwicklung intelligenter Fahrzeuge und zur Bewältigung der Verkehrssteuerung wird im Wege der Telematik eine ganz besonders hohe Erwartungshaltung zur Lösung der anstehenden Probleme entgegen gebracht. Telematik - das ist die Zusammenführung der Begriffe TELEkommunikation, AutoMAtion und InformaTIK - ist eine technische Disziplin, die in neuen Anwendungsgebieten im Verkehr und Transport wesentliche technische Fortschritte im System Fahrzeug-Infrastruktur bewirken wird.

Der Schwerpunkt der vorliegenden Ausgabe liegt auf verkehrsspezifischen Themen, in welchen dem Einsatz der Telematik, im zuvor definierten Sinne, ein besonderes Potenzial bei der Lösung anstehender Probleme wie der Sicherheit, der Effizienz und der Qualität des Verkehrs zugesprochen wird. In diesem Kontext hat sich in den letzten zehn Jahren weltweit der

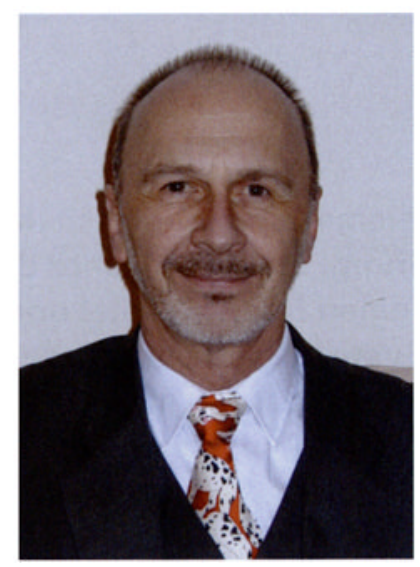

Begriff ITS - Intelligent Transport Systems - etabliert, unter welchem Investitionen in Forschung und Entwicklung für Fahrzeuge, Antriebssysteme, Verkehrsinfrastruktur und Verkehrssteuerung in Milliardenhöhe in den nächsten 20 Jahren erwartet werden.

Die Auswahl der Beiträge zeigt nur ansatzweise das weite Feld der Einsatzmöglichkeiten der Informationstechnik und der Telematik im Anwendungsbereich Verkehr sowohl fahrzeugseitig als auch für Verkehrsplanung, Verkehrsinformation und Verkehrssteuerung auf.

Hier liegt ein zukunftsträchtiges Betätigungsfeld für Wissenschaft, Industrie und Gewerbe vor uns - sowohl im Bereich der Grundlagenforschung als auch in der industriellen Umsetzung, welche als außerordentliche Chance für universitäre Forschung, die Industrie als auch für den Wirtschaftsstandort Österreich gesehen werden kann.

Ich wünsche den geneigten Leserinnen und Lesern viel Vergnügen beim Studium der Beiträge und hoffe, dass daraus eine Vielzahl von Anregungen und Innovationen in ihrem eigenen beruflichen Umfeld initiiert werden kann.

\section{Dr. Reinhard PfliegI}

via-donau

Donau Transport Entwicklungsgesellschaft $\mathrm{mbH}$ 